\begin{tabular}{|c|c|}
\hline Title & Further enhancement of the near-field on A u nanogap dimers using quasi-dark plasmon modes \\
\hline Author(s) & Shibata, Kizuku; Fujii, Sho; Sun, Quan; Miura, A tsushi; Ueno, Kosei \\
\hline Citation & $\begin{array}{l}\text { Journal of chemical physics, 152(10), } 104706 \\
\text { https://doi.org/10.1063/.5142569 }\end{array}$ \\
\hline Issue Date & 2020-03-14 \\
\hline DOC URL & http:/hdl.handle.net/2115/80589 \\
\hline Rights & $\begin{array}{l}\text { This article may be downloaded for personal use only. A ny other use requires prior permission of the author and AIP } \\
\text { Publishing. This article appeared in (citation of published article) and may be found at } \\
\text { https://aip.scitation.org/doi/10.1063/1.5142569 }\end{array}$ \\
\hline Type & article \\
\hline File Information & J. Chem. Phys.152-10_104706.pdf \\
\hline
\end{tabular}

Instructions for use 


\section{Further enhancement of the near-field on Au nanogap dimers using quasi-dark plasmon modes ${ }^{\circ}$}

Cite as: J. Chem. Phys. 152, 104706 (2020); https://doi.org/10.1063/1.5142569

Submitted: 16 December 2019 . Accepted: 20 February 2020 . Published Online: 09 March 2020

Kizuku Shibata, Sho Fujii (D), Quan Sun (D), Atsushi Miura (D), and Kosei Ueno (D)

\section{COLLECTIONS}

Paper published as part of the special topic on Collection

F This paper was selected as Featured

\section{ARTICLES YOU MAY BE INTERESTED IN}

On factors limiting the performance of photoelectrochemical $\mathrm{CO}_{2}$ reduction

The Journal of Chemical Physics 152, 100901 (2020); https://doi.org/10.1063/1.5141390

Interfacial solvation can explain attraction between like-charged objects in aqueous solution

The Journal of Chemical Physics 152, 104713 (2020); https://doi.org/10.1063/1.5141346

Enhancement of the second harmonic signal of nonlinear crystals by self-assembled gold nanoparticles

The Journal of Chemical Physics 152, 104711 (2020); https://doi.org/10.1063/1.5139893
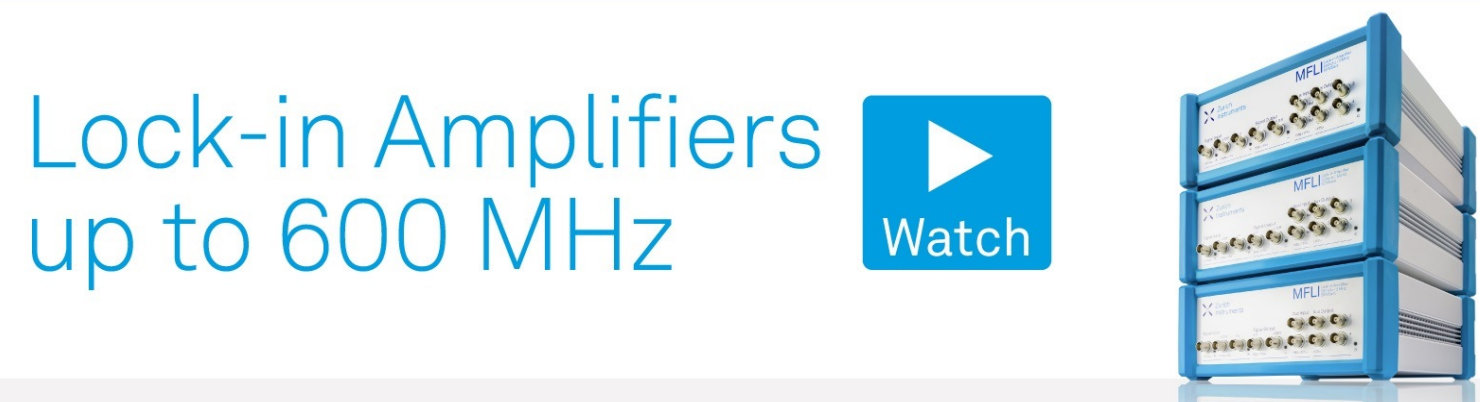

J. Chem. Phys. 152, 104706 (2020); https://doi.org/10.1063/1.5142569

152,104706

(c) 2020 Author(s) 


\title{
Further enhancement of the near-field on Au nanogap dimers using quasi-dark plasmon modes
}

\author{
Cite as: J. Chem. Phys. 152, 104706 (2020); doi: 10.1063/1.5142569 \\ Submitted: 16 December 2019 - Accepted: 20 February 2020 • \\ Published Online: 9 March 2020
}

\author{
Kizuku Shibata, Sho Fujii, (D) Quan Sun, (D) Atsushi Miura, (D) and Kosei Ueno ${ }^{\text {a) }}$ (D) \\ AFFILIATIONS \\ Department of Chemistry, Faculty of Science, Hokkaido University, Kita 10, Nishi 8, Kita-ku, Sapporo, Hokkaido 060-0810, Japan
}

\author{
a) Author to whom correspondence should be addressed: ueno@sci.hokudai.ac.jp
}

\begin{abstract}
Metallic nanogap dimers are extremely useful for enhancing surface-enhanced Raman scattering and various nonlinear optical effects employing near-field enhancement effects induced by the localized surface plasmon resonance. However, the metallic nanogap dimers exhibit an intense light scattering due to the strong dipole-dipole interaction between two metallic nanostructures and, therefore, are not necessarily a structural design that exhibits the highest near-field enhancement due to the radiation loss. Here, we propose further enhancement of the near-field on metallic nanogap dimers using quasi-dark plasmon modes. By coupling with gold ( $\mathrm{Au}$ ) nanorods having the same plasmon resonant wavelength, but completely different sizes, a quasi-dark plasmon mode, which reduces the radiation loss slightly, is induced, resulting in the elongation of the plasmon dephasing time. As a result, the signal of surface-enhanced Raman scattering of crystal violet molecules adsorbed on the Au nanogap dimer is enhanced up to about three times as compared to that measured using the Au nanogap dimer without the Au nanorods. Scattering spectrum measurements as well as electromagnetic simulations were performed to clarify the mechanism for further enhancement of the near-field. The proposed coupled plasmonic system is expected to be advantageous, especially in enhancing nonlinear optical effects using plasmonic enhancement effects.
\end{abstract}

Published under license by AIP Publishing. https://doi.org/10.1063/1.5142569

\section{INTRODUCTION}

Nanoparticles of noble metals such as gold $(\mathrm{Au})$ and silver $(\mathrm{Ag})$ exhibit localized surface plasmon resonances (LSPRs). LSPRs are collective oscillations of conduction electrons of metallic nanoparticles induced by the electric field of incident light. ${ }^{1}$ LSPRs allow metallic nanoparticles to absorb and scatter light of a certain wavelength depending on the size and the shape of metallic nanoparticles. $^{2-5}$ LSPRs show near-field enhancement effects that are several orders of magnitude higher than the incident light field in the vicinity of metallic nanoparticles. ${ }^{6-8}$ Light can be confined in the metallic nanoparticles as a near-field for a certain time $(\sim \mathrm{fs})$ because the coherent electronic oscillation continues after the incident light passes and the near-field is localized at a nanometer-sized volume like focusing by a super-lens that can break the diffraction limit. Therefore, temporal and spatial effects play a key role in the near-field enhancement. Namely, LSPRs can be regarded as a kind of nano-optical resonator.
$Q / V$ is known as a parameter exhibiting the intensity of light in the resonator. $Q$ is related to the quality factor of resonances, that is, concerning how long light is confined. In the case of LSPRs, it is related to phase relaxation time (dephasing), that is, when the coherent electronic oscillations fall apart. $V$ is related to the mode volume, that is, how small the near-field can be localized. Nanogap metallic structures such as bow-tie antennae, ${ }^{9}$ Au nanogap dimers, ${ }^{10}$ and nanogap resonators ${ }^{11}$ have been considered to show the highest near-field enhancement effect so far. At the nanogap, it can be estimated that the near-field is enhanced by $10000-100000$ times as compared to the incident light intensity, and a single molecule detection based on the surface-enhanced Raman scattering (SERS) has been reported. ${ }^{12-15}$ However, it has been revealed that the resonance $Q$ value is reduced by shortening the dephasing time since nanogap metallic structures exhibit intense light scattering due to the strong dipole-dipole interaction between structures (in-phase mode). ${ }^{16}$ Therefore, the structure showing the highest near-field enhancement is not necessarily a metallic nanogap dimer, and not 
only a mode volume, but also a quality factor is taken into consideration for the structural design to obtain an extremely strong near-field enhancement.

Coupled plasmonic systems such as a plasmon hybridization, ${ }^{17,18}$ a modal strong coupling, ${ }^{19,20}$ and a dark plasmon ${ }^{21,22}$ have received considerable attention as a tool for harvesting light efficiently with a wider spectral range or elongating the plasmon dephasing time. In particular, the modal strong coupling with optical modes having a high $Q$ value as well as the excitation of dark plasmon modes such as quadrupole and out-of-phase plasmon modes is promising for elongating the plasmon dephasing time. ${ }^{20,23}$ Recently, we have successfully elucidated that the elongation of the plasmon dephasing time results in an improvement of the near-field enhancement by exciting an out-of-phase plasmon mode of the metalinsulator-metal metamaterial nanostructures. ${ }^{24}$ In a simple design of a metal-insulator-metal nanostructure, however, the mode volume $(V)$ does not become small because the near-field localization effect is small due to the wide area of the nanogap region, whereas the $Q$ value due to the dark plasmon excitation can be increased. ${ }^{24}$ On the other hand, multimer structures such as a heptamer with multiple nanogaps can also induce the out-of-phase plasmon mode. ${ }^{25,26}$ However, multiple nanogaps also diminish the near-field localization effects. ${ }^{27}$ Therefore, it is difficult to localize the near-field at a tiny space in order to reduce the radiation loss by the dark plasmon mode, which seems to be a trade-off relationship.

In the present study, a quasi-dark plasmon mode is induced by dispersing Au nanorods whose plasmon resonant wavelength completely accords with that of Au nanogap dimers and whose size and shape are apparently different from that of Au nanogap dimers. By placing the extremely small $\mathrm{Au}$ nanorods on the $\mathrm{Au}$ nanogap dimer via a $\sim \mathrm{nm}$-sized spacer using a surfactant as a surrounding of the Au nanorod, a quasi-dark plasmon mode can be induced. The quasi-dark plasmon mode is caused by the strong nearfield interaction between the Au nanogap dimer and $\mathrm{Au}$ nanorods and has a characteristic of a relatively longer dephasing time than that of the Au nanogap dimer. The near-field enhancement is improved by the elongation of the plasmon dephasing time due to the excitation of the quasi-dark plasmon mode while maintaining the smaller nanogap width. SERS is used for the evaluation of the near-field enhancement in the proposed nanostructures in this study. This research asserts that it will lead to design guidelines for all chemical reactions and sensors using the plasmonic enhancement.

\section{EXPERIMENTAL}

\section{A. Materials}

All reagents were used as received without purification. An electron beam resist (ZEP-520a), the resist thinner (ZAP-A), the developer (ZED-N50), and the resist remover (ZDMAC) were purchased from Zeon Co. Acetone, methanol, hexadecyltrimethylammonium bromide (CTAB), hydrogen tetrachloroaurate(III) trihydrate $\left(\mathrm{HAuCl}_{4} \cdot 3 \mathrm{H}_{2} \mathrm{O}\right)$, sodium borohydride $\left(\mathrm{NaBH}_{4}\right)$, silver nitrate $\left(\mathrm{AgNO}_{3}\right)$, and $\mathrm{L}(+)$-ascorbic acid were purchased from FUJIFILM Wako Pure Chemical Corp. 5-Bromosalicylic acid (BrSA) was purchased from Tokyo Chemical Industry Co.

\section{B. Fabrication of Au nanogap dimers}

Au nanogap dimers with a different gap width were fabricated by electron beam lithography and lift-off techniques. ${ }^{28} \mathrm{~A}$ microscope cover glass [Matsunami Co., $24 \mathrm{~mm} \times 24 \mathrm{~mm}$, thickness: $0.17-0.25 \mathrm{~mm}$ (No. 2)] was cleaned by immersing in acetone, methanol, and ultra-pure water under ultrasonic conditions for every 2 min. ZEP-520a diluted by ZEP-A (1:1) was spin-coated on the glass substrate under the conditions of $1000 \mathrm{rpm}$ for $5 \mathrm{~s}$ and $4000 \mathrm{rpm}$ for $90 \mathrm{~s}$. Then, the resist-coated substrate was pre-baked on the hot plate at $110^{\circ} \mathrm{C}$ for $3 \mathrm{~min}$. Electron beam lithography was performed at a dose rate of $128 \mu \mathrm{C} \mathrm{cm}^{-2}$ by an ultra-high precision electron-beam lithography system (Elionix Co., ELS-7000HM) working at $100 \mathrm{kV}$ as an acceleration voltage. The exposed substrate was then immersed in ZED-N50 for $10 \mathrm{~min}$. $\mathrm{Cr}$ and $\mathrm{Au}$ were deposited on the developed substrate by sputtering (Ulvac Co., ACD-4000-C3-HS Compact sputter system) with thicknesses of $2 \mathrm{~nm}$ and $30 \mathrm{~nm}$, respectively. Cr was used as an adhesion layer between the glass substrate and Au nanostructures. Finally, lift-off was performed to remove the excess Au-film on the resist and leave $\mathrm{Au}$ nanostructures on the substrate by acetone and ZDMAC under ultrasonic conditions for every $2 \mathrm{~min}$.

\section{Preparation of Au nanorods}

$\mathrm{Au}$ nanorods were prepared by the seed-mediated method using CTAB worm-like micelles as a template. ${ }^{2,4,29,30}$ The seed solution was prepared as described in the following method. A $5 \mathrm{ml}$ of $\mathrm{HAuCl}_{4}$ aqueous solution $(0.5 \mathrm{mmol} / \mathrm{l})$ was mixed with $5 \mathrm{ml}$ of CTAB solution $(0.2 \mathrm{mmol} / \mathrm{l}) .^{30,31}$ A $1 \mathrm{ml}$ of $\mathrm{NaBH}_{4}$ solution $(6 \mathrm{mmol} / \mathrm{l})$ was then added to the $\mathrm{HAuCl}_{4} / \mathrm{CTAB}$ solution under stirring. On the other hand, in the growth solution, the CTAB/BrSA system was used for reducing the micelle-forming surfactants and the aging time with a high tunability of the aspect ratio. ${ }^{31}$ A $50 \mathrm{ml}$ of CTAB solution $(0.1 \mathrm{mmol} / \mathrm{l})$ together with $0.22 \mathrm{~g}$ of $\mathrm{BrSA}$ was mixed with $2.4 \mathrm{ml}$ of $\mathrm{AgNO}_{3}$ solution ( $4 \mathrm{mmol} / \mathrm{l}$ ). A $50 \mathrm{ml}$ of $\mathrm{HAuCl}_{4}$ solution $(1 \mathrm{mmol} / \mathrm{l})$ was then added to the $\mathrm{CTAB} / \mathrm{BrSA} / \mathrm{AgNO}_{3}$ solution. After slow stirring for a while, a $0.4 \mathrm{ml}$ of ascorbic acid solution (64 mmol/l) was added. To produce Au nanorods, a $24 \mathrm{ml}$ of the growth solution was mixed with $0.8 \mathrm{ml}$ of the seed solution at a temperature of $30^{\circ} \mathrm{C}$ for $12 \mathrm{~h} .{ }^{31}$ The centrifugations were performed with $\sim 2000 \mathrm{~g}$ to remove the excess CTAB in the solution several times for $30 \mathrm{~min}$.

\section{Spectral properties of Au nanogap dimers with and without Au nanorods}

Extinction spectra of the Au nanogap dimers with and without Au nanorods were measured by absorption microspectroscopy. The Au nanogap dimers with Au nanorods (Au nanorods/Au nanogap dimer) were prepared by dropping a $2 \mu \mathrm{l}$ of the Au nanorods solution on the substrate of the Au nanogap dimer and the substrate was air-dried. The halogen light equipped with an inverted optical microscope (Olympus Co., IX-71) was used as the light source for the measurement. Light passed through the long working distance condenser lens (Olympus Co., IX-ULWCD, NA = 0.3) and the sample substrate was collected by an objective lens (Olympus Co., LUCPLFLN60X, NA = 0.7), and then the probe light was introduced into the spectrometer (Princeton Instruments, SP-2358/PIXIS: 100BR) 
via a pinhole with a diameter of $400 \mu \mathrm{m}$. The number of the $\mathrm{Au}$ nanogap dimers in the measured area is estimated to be $\sim 220$. A linear polarizer was set before the condenser lens. The reference of the extinction spectrum measurement $\left(I_{0}\right)$ was obtained by focusing on the glass substrate, and the sample $(I)$ was measured by focusing on the Au nanogap dimers. After the measurement, the extinction spectrum was obtained by calculating $-\log \left(I / I_{0}\right)$ for every wavelength. The scattering spectra were measured by using a high NA dark field condenser lens (Olympus Co., Universal Condenser U-UCD8, $\mathrm{NA}=0.8$ ). The other optical setup is the same as that for extinction spectrum measurement.

\section{E. Surface-enhanced Raman scattering measurements of crystal violet molecules}

A $2 \mu \mathrm{l}$ of crystal violet aqueous solution $\left(5.0 \times 10^{-4} \mathrm{~mol} / \mathrm{l}\right)$ was dropped and kept stationary for $1 \mathrm{~min}$. Then, the substrate was cleaned by immersing in ultra-pure water under ultrasonic conditions to remove the excess crystal violet molecules on the substrate. The Raman microspectroscopic system (RENISHAW, inVia Reflex) was used for the measurement of Raman scattering spectra. The laser wavelength and the objective lens used in this study were $785 \mathrm{~nm}$ and $50 \times$ (Zeiss, NA 0.75 ), respectively. The laser power of $136 \mu \mathrm{W}$ was chosen and the exposure time was set as $1 \mathrm{~s}$.

\section{F. Finite-difference time-domain simulations}

Electromagnetic simulations were performed to elucidate the far-field and near-field spectra and the near-field intensity distribution in temporal and spatial domains by a Finite-Difference TimeDomain (FDTD) method using the FDTD Solutions software package (Lumerical, Inc.). The optical properties of $\mathrm{Au}$ were obtained using the data from Johnson and Christy. ${ }^{32}$ The FDTD simulations were performed on a discrete, uniformly spaced mesh with a mesh size of $1 \mathrm{~nm}$. The total-field scattered-field (TFSF) source was injected into the Au nanorod covered with a $2 \mathrm{~nm}$-thick dielectric layer with a refractive index of 1.4 assuming $\mathrm{CTAB}$ and the $\mathrm{Au}$ nanogap dimer from the structure side.

\section{RESULTS AND DISCUSSIONS}

\section{A. Far-field spectral properties of Au nanogap dimers with and without Au nanorods}

A scanning electron microscope (SEM) image of Au nanogap dimers with a designed gap width of $6 \mathrm{~nm}$ is shown in Fig. 1(a). The size of each Au nanoblock is $100 \mathrm{~nm} \times 100 \mathrm{~nm} \times 30 \mathrm{~nm}$, and the structures are diagonally aligned via a nanogap. The nanogap width is defined as a distance from edge to edge. Extinction spectra of Au nanogap dimers with a different gap width under the incident polarization parallel to the $x$-axis are shown in Fig. 1(b). The inset in Fig. 1(b) shows a definition of $x$ - and $y$-axes to the Au nanogap dimer. The LSPR band shows an obvious red-shift with the decreasing nanogap width due to the dipole-dipole interaction between two nanoblock structures. ${ }^{33}$ Under the incident polarization parallel to $y$-axis, on the other hand, the LSPR band does not change at around $670 \mathrm{~nm}$ with changing the nanogap width (data are not shown here) because the near-field interaction is quite small for the polarization.
Figure 1(c) exhibits an extinction spectrum of the solution in which Au nanorods are dispersed. The transverse LSPR band is peaking at a wavelength of $520 \mathrm{~nm}$, while the longitudinal LSPR band is peaking at a wavelength of $818 \mathrm{~nm}$. Therefore, the LSPR bands of both the Au nanogap dimer and the Au nanorods (longitudinal mode) might overlap with each other in the dried system due to the surrounding reflective index change. Figure 1(d) shows extinction spectra of the Au nanogap dimer and the $\mathrm{Au}$ nanorods/Au nanogap dimer with a gap width of $6 \mathrm{~nm}$. A distinct longer wavelength shift was confirmed, and a slight spectrum dip could be seen in the spectrum at the resonant wavelength of Au nanorods in the dried system, whose resonant wavelength was extrapolated by the FDTD simulation based on the experimental spectrum in an aqueous solution. Figure 1(e) shows a typical SEM image of the structure, in which the Au nanorod is bridged above the nanogap dimer. On the other hand, Fig. 1(f) indicates typical SEM images of Au nanorods located near the nanogap region. Because the Au nanorod is covered with a CTAB surfactant, some positions in the SEM image have become brighter due to charge-up. The yield of the structure in which the $\mathrm{Au}$ nanorod is bridged above the nanogap dimer is estimated to be $\sim 10 \%$, whereas the structure in which Au nanorods are located near the nanogap is $\sim 75 \%$. The remaining $\sim 15 \%$ did not have Au nanorods near the nanogap or were difficult to distinguish due to the charge-up. It is considered that most $\mathrm{Au}$ nanorods are located near the nanogap region because the $\mathrm{Au}$ nanorods might be dammed near the nanogap region during the evaporation process.

\section{B. SERS properties of Au nanogap dimers with and without Au nanorods}

Figure 2(a) shows Raman scattering spectra of crystal violet molecules immobilized on the Au nanogap dimers with a different nanogap width. The crystal violet was immobilized on the surface of $\mathrm{Au}$ nanostructures through a coordinate bond between the lone pair of nitrogen in the crystal violet and Au surface. From Langmuir's adsorption isotherm, it has been confirmed in advance that it is an adsorption saturation condition. ${ }^{34}$ Namely, the crystal violet molecules are homogeneously immobilized only on the $\mathrm{Au}$ surface due to the adsorption saturation and the powerful cleaning with ultra-pure water. It is clearly demonstrated from Fig. 2(a) that the SERS intensity apparently increased with a decrease in the nanogap width. This result is attributed not only to an induction of the strong near-field enhancement accompanying with a decrease in the nanogap width, but also to the overlapping of the excitation laser and Raman scattering wavelengths with the plasmon resonance band of the structures having a smaller nanogap width.

On the other hand, Raman scattering spectra of crystal violet molecules immobilized on the Au nanorods/Au nanogap dimer with different nanogap widths are shown in Fig. 2(b). SERS intensity increased significantly at any gap width in the Au nanorods/Au nanogap dimers. In particular, the SERS intensity of the nanostructures with gap widths of $6 \mathrm{~nm}, 8 \mathrm{~nm}$, and $11 \mathrm{~nm}$ are extraordinarily enhanced. It is noteworthy that the Raman scattering intensity on $\mathrm{Au}$ nanorods only deposited on the glass substrate is lower than that with a gap width of $142 \mathrm{~nm}$ shown in Fig. 2(b) (data are not shown here). From the results, the placement of $\mathrm{Au}$ nanorods on the $\mathrm{Au}$ 
(a)

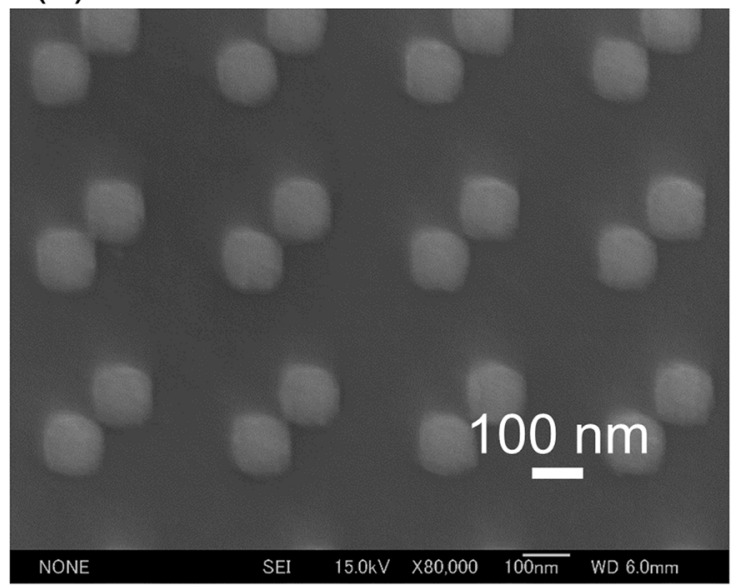

(c)

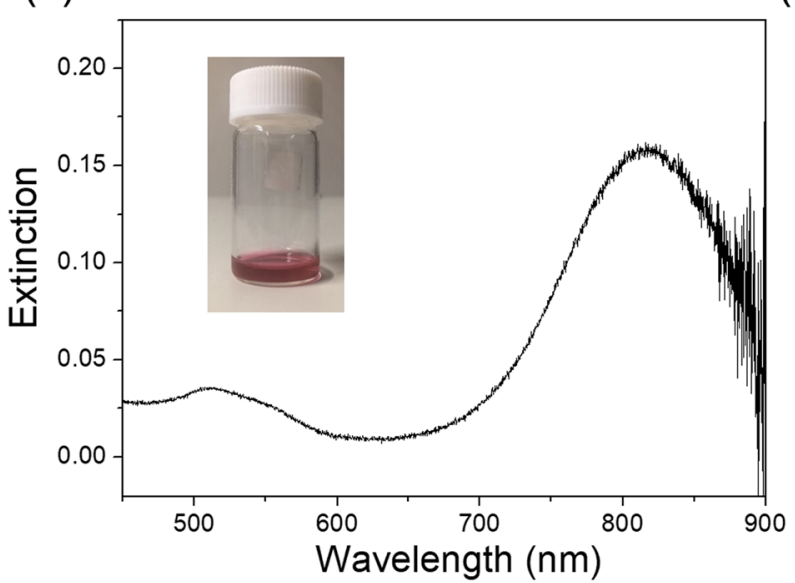

(e)

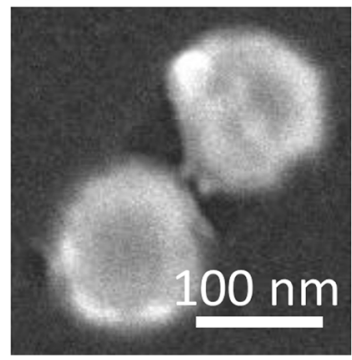

(b)

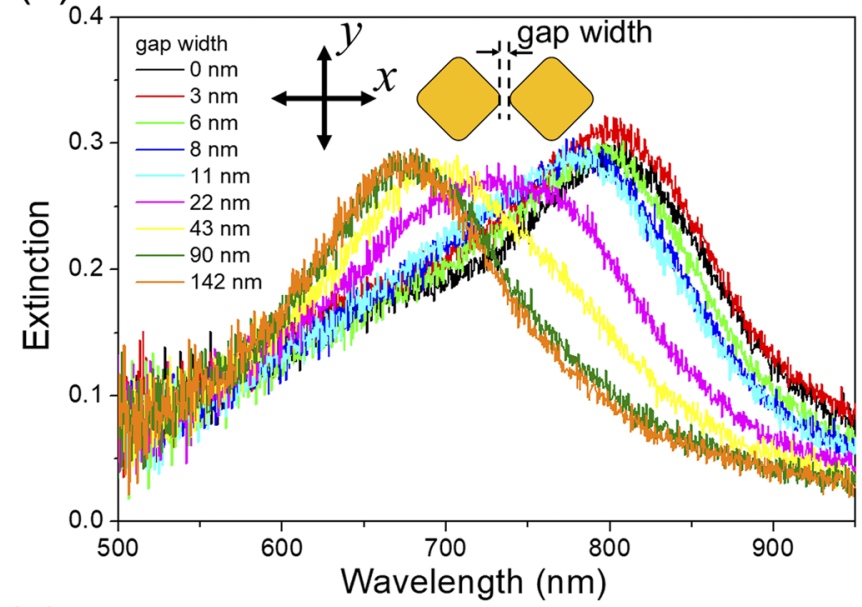

(d)
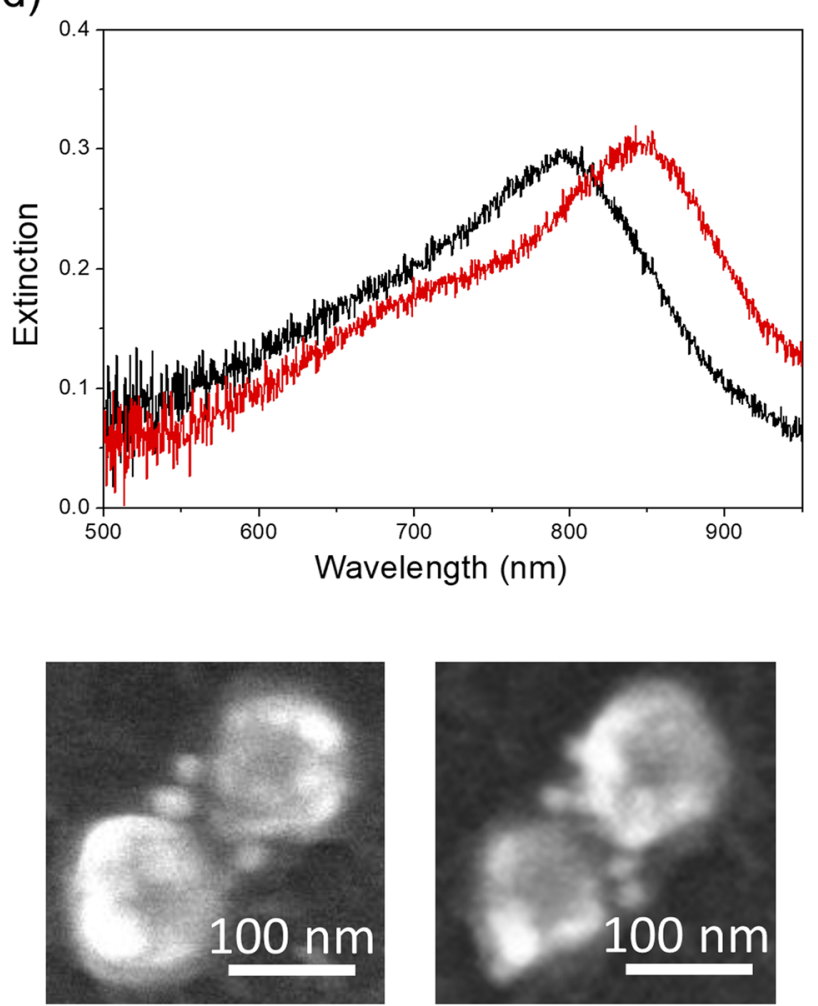

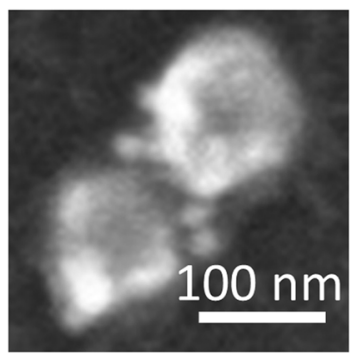

FIG. 1. (a) A SEM image of Au nanogap dimers with a gap width of $6 \mathrm{~nm}$. (b) Extinction spectra of Au nanogap dimers with a different gap width under the incident polarization parallel to $x$-axis. The inset in (b) shows a definition of $x$ - and $y$-axes to the Au nanogap dimer. (c) An extinction spectrum of the solution in which Au nanorods are dispersed. The inset shows a picture of the solution. (d) Extinction spectra of the Au nanogap dimer (black curve) and the Au nanorods/Au nanogap dimer (red curve) with a gap width of $6 \mathrm{~nm}$. (e) A typical SEM image of the structure in which the Au nanorod is bridged above the nanogap dimer. (f) Typical SEM images of the structures in which Au nanorods are located near the nanogap region.

nanogap dimers plays an important role in the further enhancement of SERS on the Au nanogap dimer.

Figure 3(a) shows gap width dependences of SERS intensity at $1179 \mathrm{~cm}^{-1}$, which is corresponding to $\mathrm{C}$-phenyl, $\mathrm{C}-\mathrm{H}$ in-plane antisymmetric stretching mode measured using the Au nanogap dimer with and without Au nanorods. ${ }^{36}$ Comparing the maximum values, it can be seen clearly that the SERS signal measured by the $\mathrm{Au}$ nanorods/ $\mathrm{Au}$ nanogap dimer is about 3 times larger than that 
(a)

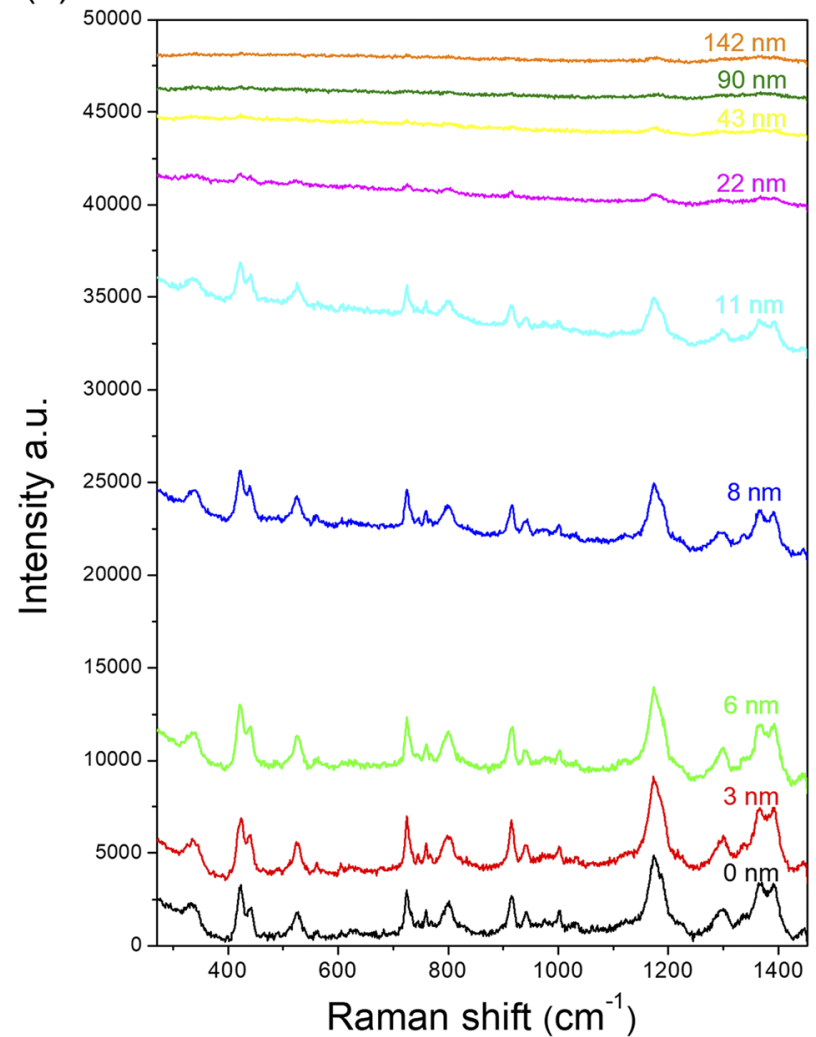

(b)

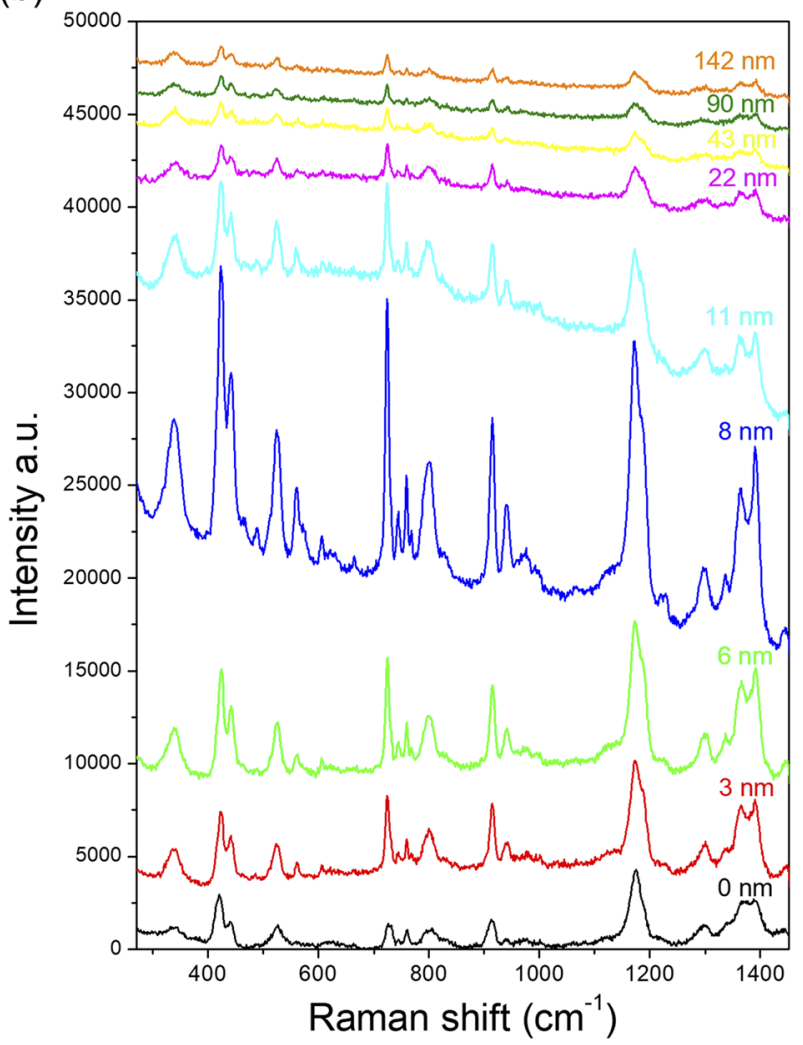

FIG. 2. (a) Raman scattering spectra of crystal violet molecules immobilized on the Au nanogap dimers with different nanogap widths without Au nanorods. (b) Raman scattering spectra of crystal violet molecules immobilized on the Au nanorods/Au nanogap dimer with different nanogap widths. The inset figures show the designed gap width.

(a)

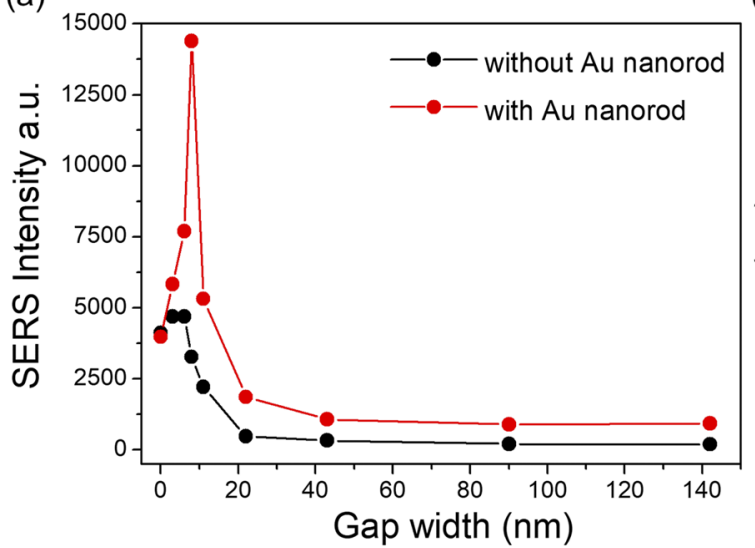

(b)

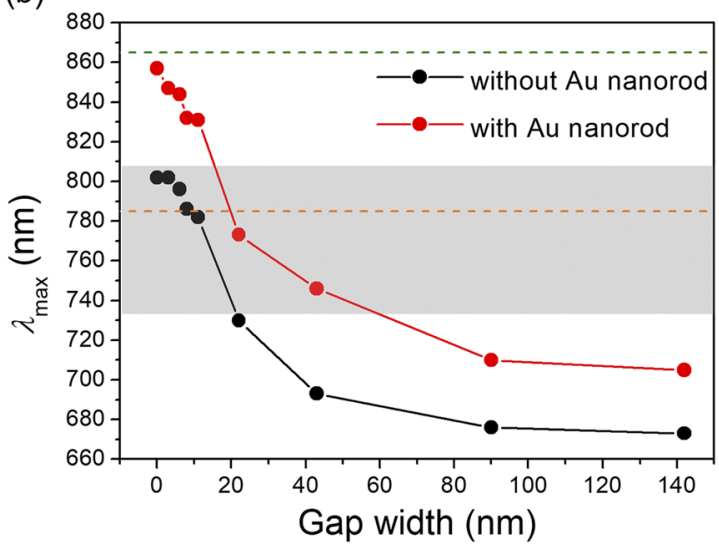

FIG. 3. (a) Gap width dependences of SERS intensity at $1179 \mathrm{~cm}^{-1}$ measured using the Au nanogap dimer with and without Au nanorods. (b) Gap width dependences of the LSPR peak wavelength of the Au nanogap dimer with and without Au nanorods. The data were obtained from the extinction spectra shown in Fig. 1 (b) and its corresponding figure with Au nanorods including Fig. 1(d). The dip wavelength centered around $770 \mathrm{~nm}$ is highlighted by a gray color band. The excitation wavelength and Raman scattering wavelength which correspond to $1179 \mathrm{~cm}^{-1}$ are indicated by orange and green broken lines, respectively. 
of the Au nanogap dimer without Au nanorods. The distribution of the structural size and the gap width fabricated by the same method should be $\sim 3 \mathrm{~nm}$ as a standard deviation from the previous study. ${ }^{37,38}$ However, the average near-field enhancement effect of the ensembled Au nanogap dimers showed a clear gap width dependence even by the change of $1 \mathrm{~nm} .^{37}$ The gap width dependence of SERS from the Au nanorods/Au nanogap dimer has a high reproducibility and is reliable to some extent as an ensemble averaged measurement although Au nanorods are dispersed randomly. It is noteworthy that the maximum enhancement of SERS was obtained not at a gap width of $3 \mathrm{~nm}$ but at a gap width of around $8 \mathrm{~nm}$.

Figure 3(b) shows gap width dependences of the LSPR peak wavelength of the Au nanogap dimer with and without Au nanorods, whose data were obtained from these extinction spectra shown in Fig. 1(b) and its corresponding figure with Au nanorods. Both the gap width dependences with and without $\mathrm{Au}$ nanorods show a longer wavelength shift with the decreasing gap width due to the dipole-dipole interaction. The further spectrum shift can be seen in the presence of $\mathrm{Au}$ nanorods. Importantly, the dip wavelength centered around $770 \mathrm{~nm}$ which should be the peak wavelength of $\mathrm{Au}$ nanorods in the dried system overlaps with the plasmon resonance spectrum of the Au nanogap dimers with gap widths from $0 \mathrm{~nm}$ to $12 \mathrm{~nm}$. Namely, a stronger near-field interaction between LSPRs of the Au nanogap dimer and the Au nanorod can be expected with gap widths from $0 \mathrm{~nm}$ to $12 \mathrm{~nm}$.

On the other hand, the excitation wavelength $(785 \mathrm{~nm})$ for the SERS measurement and the Raman scattering wavelength $(865 \mathrm{~nm})$ which corresponds to $1179 \mathrm{~cm}^{-1}$ are highlighted in Fig. 3(b) as broken lines. From the electromagnetic theory in SERS, 39,40 SERS intensity is related to the near-field enhancement effect at both wavelengths of the incident light as well as the scattering light, based on the following equation:

$$
P_{s}\left(v_{s}\right)=N \sigma_{S E R S} L\left(v_{i}\right)^{2} L\left(v_{s}\right)^{2} I\left(v_{i}\right),
$$

where $L\left(v_{i}\right)^{2}$ is the enhancement factor at the wavelength of incident light $\left[L\left(v_{i}\right)=\left|E_{l o c}\left(v_{i}\right)\right| /\left|E\left(v_{0}\right)\right|\right]$, and $L\left(v_{s}\right)^{2}$ is the enhancement factor at the Raman scattering wavelength $\left[L\left(v_{s}\right)=\left|E_{l o c}\left(v_{s}\right)\right| /\left|E\left(v_{0}\right)\right|\right] .{ }^{4}$ $N$ is the number of molecules contributing to SERS signals, $\sigma_{S E R S}$ is the Raman scattering cross section including chemical effects on SERS, and $I\left(v_{i}\right)$ is the incident laser power in the SERS measurement. The peak wavelengths of extinction spectra of the $\mathrm{Au}$ nanorods/Au nanogap dimers in which the gap width ranges from $0 \mathrm{~nm}$ to $12 \mathrm{~nm}$ are between the excitation wavelength and the Raman scattering wavelength as shown in Fig. 3(b). In particular, the $\mathrm{Au}$ nanogap dimer having a gap width of $8 \mathrm{~nm}$ or $12 \mathrm{~nm}$ is considered to exhibit the highest enhancement since the stronger SERS intensity is obtained when the LSPR wavelength exists in the middle of the two wavelengths. ${ }^{4}$

On the whole, there are two possibilities for the further enhancement by placing Au nanorods as follows: (1) a stronger nearfield interaction between the Au nanogap dimer and $\mathrm{Au}$ nanorods is induced when the gap width ranges from $0 \mathrm{~nm}$ to $12 \mathrm{~nm}$ because both the LSPR bands overlap with each other, and there is a possibility that the dark plasmon mode having a longer dephasing time is induced like in a metal-insulator-metal structure. In this case, CTAB works as an insulator layer having a $\sim$ nm gap width. (2) The Au nanorods/Au nanogap dimer with a gap width of $8 \mathrm{~nm}$ or $12 \mathrm{~nm}$ is strongly resonant with incident and Raman scattering light to evolve the SERS efficiently. In order to elucidate which effect contributes to the further enhancement of SERS in this study, scattering spectrum measurements and electromagnetic simulations of the $\mathrm{Au}$ nanogap dimer with and without Au nanorods were performed.

\section{Scattering spectral properties of the Au nanogap dimer with and without Au nanorods}

Figure 4(a) indicates scattering spectra of the $\mathrm{Au}$ nanogap dimer and the $\mathrm{Au}$ nanorods/Au nanogap dimer with a gap width of $6 \mathrm{~nm}$. Notice that the spectra indicate a red-shift as compared to the extinction spectra and some other peaks are also observed because a high NA dark-field condenser lens $(\mathrm{NA}=0.8)$ was used for the measurements. The spectrum difference between extinction and scattering spectra is discussed in the supplementary material. In the case of the Au nanorods/Au nanogap dimer, however, it seems that two peaks have appeared at the relatively shorter and longer wavelengths than the peak wavelength of the Au nanogap dimer without Au nanorods. To compare the spectrum difference with and without (a)

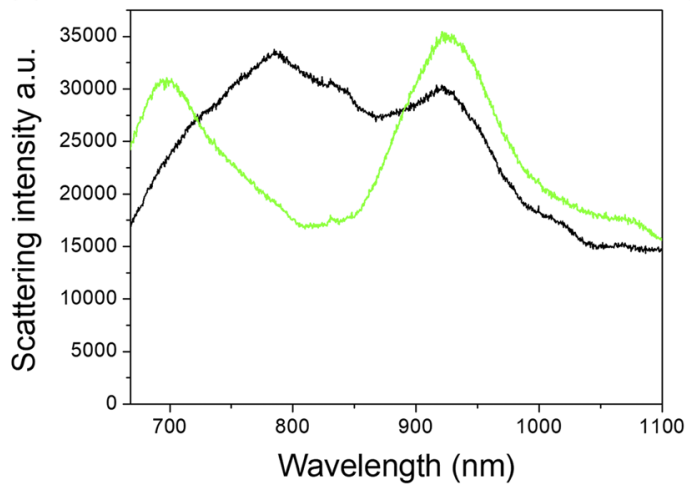

(b)

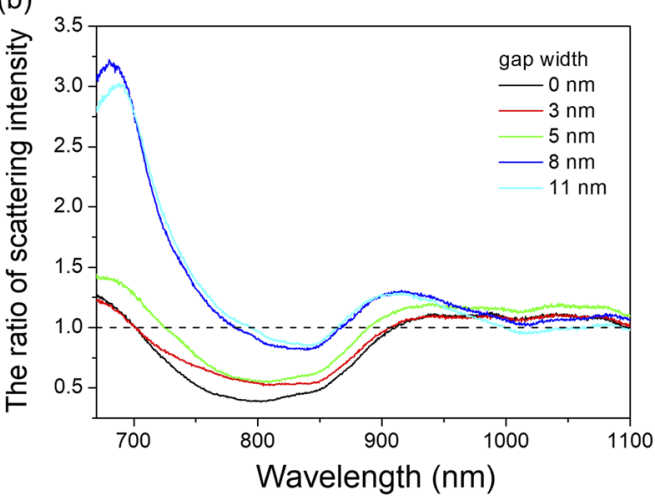

FIG. 4. (a) Scattering spectra of the Au nanogap dimer (black line) and the Au nanorods/Au nanogap dimer (green line) with a gap width of $6 \mathrm{~nm}$. (b) The ratio of the scattering spectra obtained by dividing the scattering spectrum of the Au nanorods/Au nanogap dimer by that of the Au nanogap dimer with gap widths from $0 \mathrm{~nm}$ to $11 \mathrm{~nm}$. 
Au nanorods, the ratio of the scattering spectra is obtained by dividing the scattering spectrum of the Au nanorods/Au nanogap dimer by that of the Au nanogap dimer and indicated in Fig. 4(b). When the ratio of the scattering intensity is less than 1.0, it means the suppression of scattering by placing the Au nanorods. However, it is unlikely that the Fano dip was observed by exciting the dark plasmon mode since the spectrum width of the dip is broad. Namely, it means that the plasmonic Fano resonance was not induced by the dark plasmon excitation; two peaks have appeared at the shorter and longer wavelengths of the LSPR band of the Au nanogap dimer without Au nanorods, so that it looks like a spectrum dip as a result. At both peaks, the scattering intensity of the Au nanorods/Au nanogap dimer with gap widths of $8 \mathrm{~nm}$ and $12 \mathrm{~nm}$ is slightly larger than that from $0 \mathrm{~nm}$ to $8 \mathrm{~nm}$. A kind of coupling modes are excited at both wavelengths, especially with the gap width of $8 \mathrm{~nm}$ and $11 \mathrm{~nm}$, and the scattering loss of the longer wavelength mode is smaller than the shorter wavelength mode.

\section{Numerical simulations of spectral properties and the near-field in spatial and time domains}

FDTD simulations were performed to elucidate the assignment of the coupling modes at each wavelength and the near-field in (a)

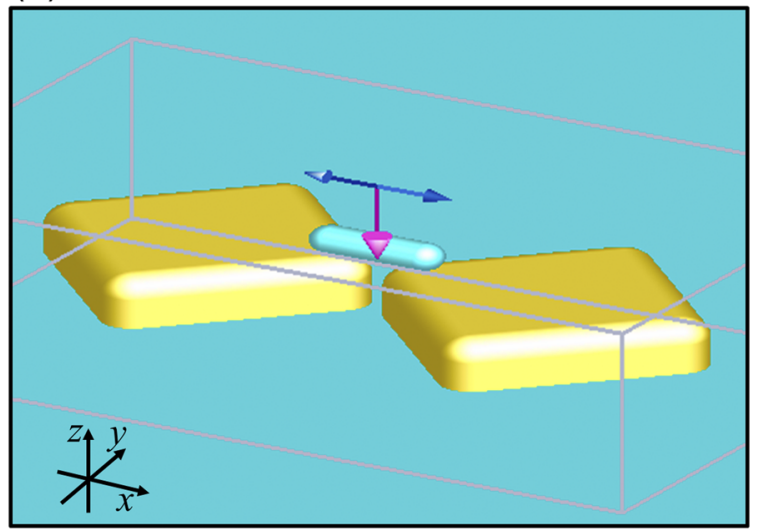

(c)

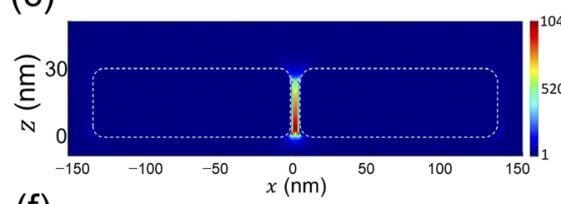

(f)

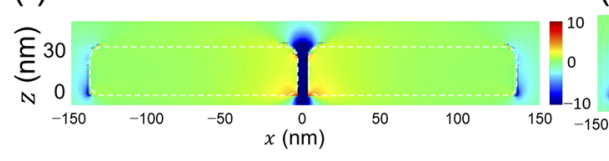

(i)

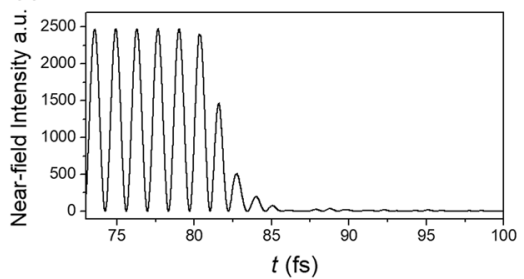

(d)

(g)
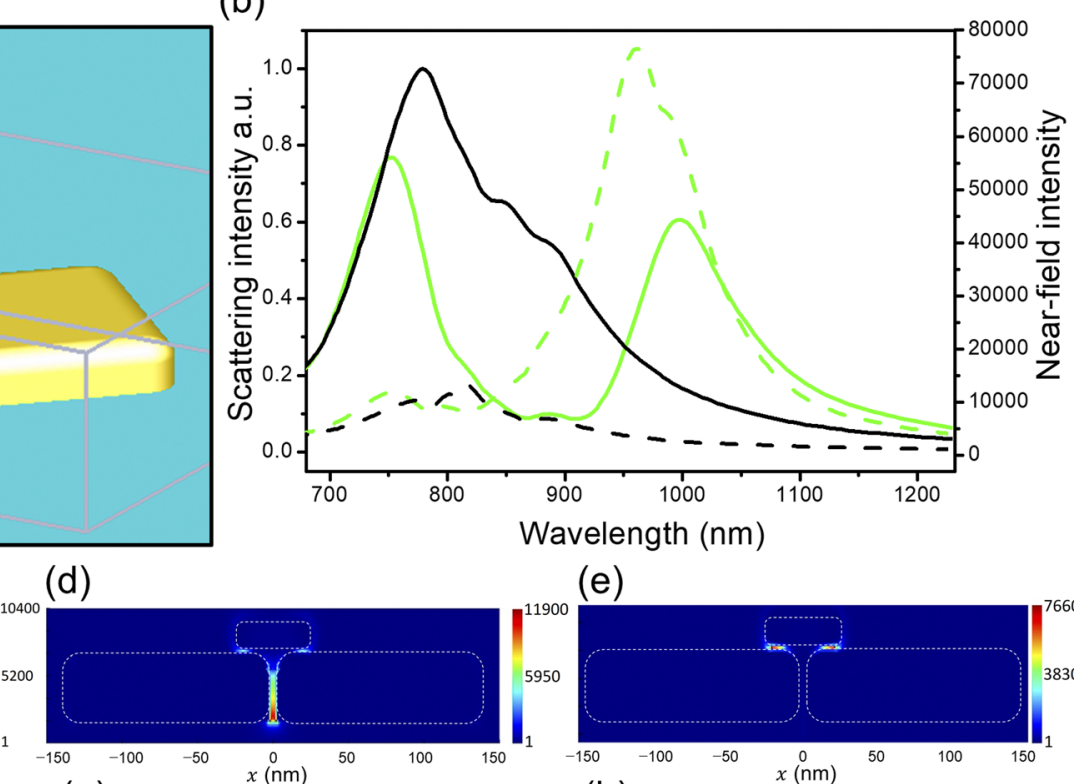

(e)

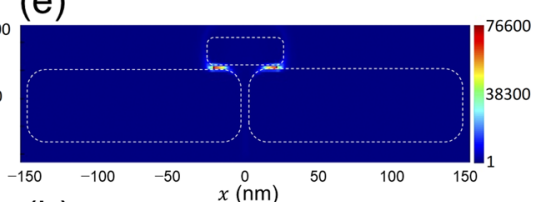

(h)

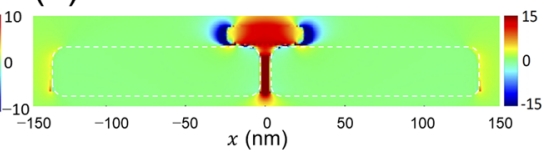

(k)
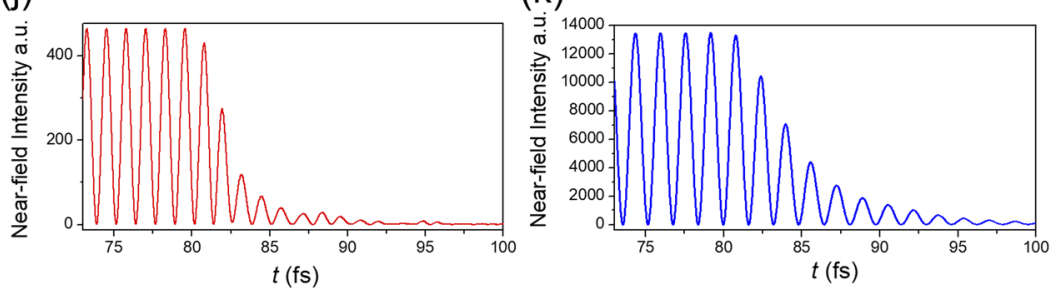

FIG. 5. (a) A schematic illustration of the FDTD simulation model with a structure in which the Au nanorod is bridged above the Au nanogap dimer. The inset arrows show the $x, y$, and $z$ coordinate axes. (b) Scattering spectra of the Au nanogap dimer (black solid line) and the structure in which the Au nanorod is bridged above the Au nanogap dimer (green solid line). Near-field spectra of the Au nanogap dimer (black broken line) and the structure in which the Au nanorod is bridged above the Au nanogap dimer (green broken line). The near-field intensity distribution on the $x-z$ plane at $y=0$ of the Au nanogap dimer at the peak wavelength (c), and the structure in which the Au nanorod is bridged above the Au nanogap dimer at the shorter peak wavelength (d) and the longer peak wavelength (e) in (b). A magnitude of the electric field along the $x$-axis by color maps on the $x-z$ plane at $y=0$ of the Au nanogap dimer at the peak wavelength $(f)$, and the structure in which the Au nanorod is bridged above the Au nanogap dimer at the shorter peak wavelength $(\mathrm{g})$ and the longer peak wavelength $(\mathrm{h})$ in $(\mathrm{b})$. The simulation results of the plasmon dephasing by monitoring the change in near-field intensity at the hot site of the Au nanogap dimer at the peak wavelength (i), and the structure in which the Au nanorod is bridged above the Au nanogap dimer at the shorter peak wavelength (j) and the longer peak wavelength $(k)$ in $(b)$. 
spatial and time domains using the simulation model as schematically illustrated in Fig. 5(a). The center of the Au nanorod was set at $x=0, y=0$, and on the top of the dimer a $2 \mathrm{~nm}$ dielectric spacer is placed parallel to the $x$-axis of the Au nanogap dimer with a gap width of $6 \mathrm{~nm}$. Scattering spectra of the Au nanogap dimer and the structure in which the Au nanorod is bridged above the Au nanogap dimer is shown in Fig. 5(b). The shape of the scattering spectra with and without Au nanorods almost reproduced the experimental results as shown in Fig. 4(a) qualitatively. There are two bands in the scattering spectrum of the structure in which the Au nanorod is bridged above the Au nanogap dimer, which are located at relatively shorter and longer wavelengths than that of the Au nanogap dimer. The corresponding near-field spectra are also shown in Fig. 5(b) indicated by broken lines. Although only the near-field spectrum of the structure in which the Au nanorod is bridged above the Au nanogap dimer is peaking at the slightly shorter wavelength of the scattering spectrum, it is the condition to induce SERS. The nearfield intensity distribution of the $x-z$ plane at $y=0$ of the Au nanogap dimer is shown in Fig. 5(c). The near-field intensity distribution was monitored at the wavelength of $790 \mathrm{~nm}$. The near-field is localized at the nanogap region and its near-field enhancement factor reaches 10400 . On the other hand, the near-field intensity distribution of the structure in which the $\mathrm{Au}$ nanorod is bridged above the $\mathrm{Au}$ nanogap dimer monitored at the shorter $(755 \mathrm{~nm})$ and the longer wavelengths $(964 \mathrm{~nm})$ are shown in Figs. 5(d) and 5(e), respectively. From the near-field intensity distribution, it is clearly elucidated that the shorter and longer wavelength peaks correspond to gap modes between the two Au nanoblocks and between the Au nanogap dimer and the Au nanorod, respectively. Note that the near-field enhancement factor of the gap mode between the Au nanogap dimer and the Au nanorod is extraordinarily enhanced as high as about 77000 although it is only about 12000 in the case of the gap mode between the two Au nanoblocks.

Figures 5(f)-5(h) show a magnitude of the electric field along the $x$-axis which indicates the phase information of LSPR by colors and corresponds to the simulation conditions as shown in Figs. 5(c)5(e), respectively, although the phase information induced at the Au nanogap dimer is almost normal, which is the same phase in each Au nanoblock. On the other hand, a quadrupole resonance is induced on the Au nanorod due to the strong near-field interaction with the local electric field at the nanogap. In particular, the gap mode between the Au nanogap dimer and the Au nanorod is influenced by the excitation of the quadrupole mode. Namely, a quasi-dark plasmon mode might be induced, especially at the longer wavelength. Figures $5(\mathrm{i})-(\mathrm{k})$ are simulation results of the plasmon dephasing which are the same monitor wavelengths and structural (a)

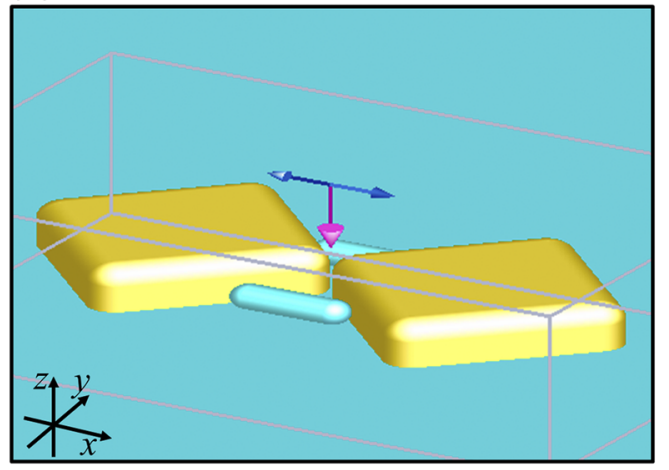

(c)

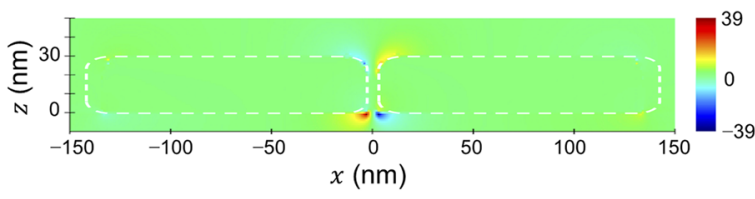

(d)

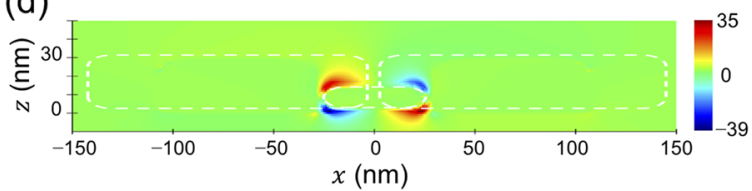

(b)

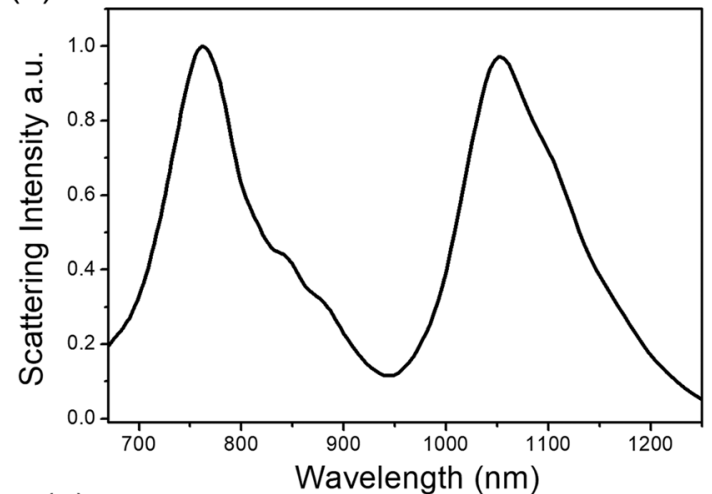

(e)

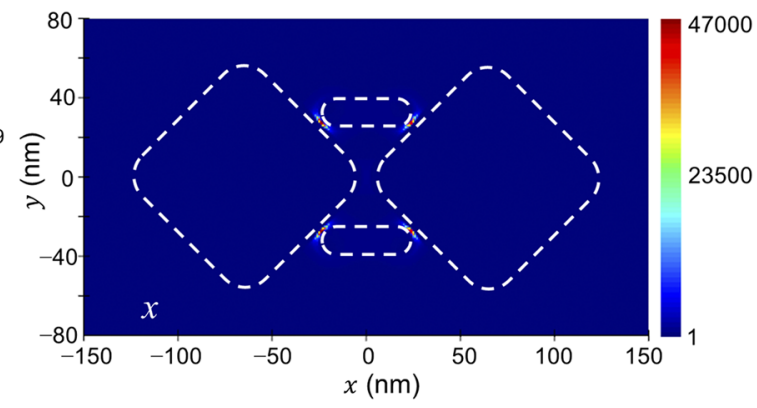

FIG. 6. (a) A schematic illustration of the FDTD simulation model with a structure in which Au nanorods are located near the nanogap region. The inset arrows show the $x, y$, and $z$ coordinate axes. (b) A simulated scattering spectrum of the structure in which Au nanorods are located near the nanogap region. (c) A phase information of the structure in which Au nanorods are located near the nanogap region indicated by color maps on the $x-z$ plane at $y=0 \mathrm{~nm}$ (at $759 \mathrm{~nm}$ wavelength). (d) The phase information of the structure in which Au nanorods are located near the nanogap region indicated by color maps on the $x-z$ plane at $y=32 \mathrm{~nm}$ (at $1017 \mathrm{~nm}$ wavelength). (e) The near-field intensity distribution on the $x-y$ plane at $z=8 \mathrm{~nm}$ (at $1047 \mathrm{~nm}$ wavelength). 
designs in Figs. 5(c)-5(e), respectively. The FDTD simulations of the plasmon dephasing were obtained by suddenly cutting the incident continuous light and monitoring the change in the near-field intensity at the hot site. From the exponential decay fitting, the lifetimes of LSPR estimated in Figs. 5(i)-5(k) are 1.2 fs, $2.1 \mathrm{fs}$, and $4.1 \mathrm{fs}$, respectively. The lifetime of LSPR of the Au nanogap dimer is very short due to the intense light scattering based on the strong dipole-dipole interaction between two nanoblocks. On the other hand, the lifetime of LSPR of the structure in which the Au nanorod is bridged above the Au nanogap dimer is elongated, especially at the longer peak wavelength in Fig. 5(b), which corresponds to the gap mode between the Au nanogap dimer and the Au nanorod. This elongation of the lifetime is thought to be due to the excitation of the quasi-dark plasmon mode and can be also understood from the fact that the scattering intensity becomes smaller despite the longer wavelength side as compared to the peak of the Au nanogap dimer.

As mentioned above, most $\mathrm{Au}$ nanorods are located near the nanogap region. Therefore, we performed FDTD simulations based on the structure in which Au nanorods are located near the nanogap region with an $\mathrm{Au}$ nanorod orientation angle of $0^{\circ}$ against the $x$-axis as shown in Fig. 6(a). Figure 6(b) shows a simulated scattering spectrum of the structure. Two distinct peaks can be seen as similar to the structure in which the $\mathrm{Au}$ nanorod is bridged above the $\mathrm{Au}$ nanogap dimer as shown in Fig. 5(b). From the phase information at the wavelengths of $759 \mathrm{~nm}$ [Fig. 6(c)] and $1017 \mathrm{~nm}$ [Fig. 6(d)], the shorter and longer wavelength peaks also correspond to gap modes between the two Au nanoblocks and between the Au nanogap dimer and the Au nanorod, respectively. Furthermore, it is noteworthy that the phase information indicates the excitation of the quasi-dark plasmon modes like a case of the structure in which the Au nanorod is bridged above the Au nanogap dimer. The near-field intensity distribution at the wavelength of $1047 \mathrm{~nm}$ is shown in Fig. 6(e). The near-field enhancement as high as 47000 is obtained at the nanogap between Au nanorods and the Au nanogap dimer, which is slightly lower than that obtained by the structure in which the $\mathrm{Au}$ nanorod is bridged above the Au nanogap dimer. FDTD simulations of the structure in which Au nanorods are located near the nanogap region with different $\mathrm{Au}$ nanorod orientation angles of $15^{\circ}$ and $30^{\circ}$ against the $x$-axis and the related discussions are described in the supplementary material. Although the near-field intensity slightly decreased from 47000 to 33600 with an increase in the orientation angle, the structure in which $\mathrm{Au}$ nanorods are located near the nanogap region with any angle still shows a strong near-field enhancement compared to just an Au nanogap dimer.

\section{CONCLUSION}

Although the metallic nanogap dimer such as a bow-tie antenna was known to be the nanostructure that shows the highest near-field enhancement based on LSPRs, the radiation loss due to the strong light scattering is fatal. In the present study, we found a methodology to improve the near-field enhancement effect by slightly reducing the radiation loss based on excitation of the quasi-dark plasmon mode while keeping the nanogap width constant. Although, as the mechanism, (1) the suppression of light scattering like a Fano dip by excitation of a dark plasmon based on the strong near-field interaction between the Au nanogap dimer and Au nanorods and (2) the enhancement effect of incident and scattering fields in SERS by a gap mode between the Au nanogap dimer and the Au nanorods were considered, the excitation of a gap mode between the Au nanogap dimer and the $\mathrm{Au}$ nanorods by the near-field interaction mainly influences the further enhancement of the near-field, which was clarified by the scattering spectrum measurements and FDTD simulations. It was concluded that the gap mode is a quasi-dark plasmon mode in which the strong light scattering is slightly suppressed, having an advantageous characteristic that the lifetime of the gap mode is as long as that of ordinary LSPR. It can be considered that the quasi-dark plasmon mode is excited by the difference in size between the Au nanogap dimer and the Au nanorod although the resonant wavelengths are same. This finding is expected to be beneficial, especially in enhancing nonlinear optical effects using plasmonic enhancement effects.

\section{SUPPLEMENTARY MATERIAL}

See the supplementary material for the comparison of simulated extinction and scattering spectra and FDTD simulation results of the structure in which $\mathrm{Au}$ nanorods are located near the nanogap region with Au nanorod orientation angles of $15^{\circ}$ and $30^{\circ}$.

\section{ACKNOWLEDGMENTS}

We acknowledge financial support from JSPS KAKENHI (Grant Nos. JP18H05205, JP19H02737, JP19H04667, JP18K18985, and JP19K15577), the Nanotechnology Platform (Hokkaido University), and the Photo-excitonix Project in Hokkaido University.

\section{REFERENCES}

${ }^{1}$ K. L. Kelly, E. Coronado, L. L. Zhao, and G. C. Schatz, J. Phys. Chem. B 107(3), 668-677 (2003).

${ }^{2}$ Y. Y. Yu, S.-S. Chang, C.-L. Lee, and C. R. C. Wang, J. Phys. Chem. B 101(34), 6661-6664 (1997).

${ }^{3}$ S. J. Oldenburg, R. D. Averitt, S. L. Westcott, and N. J. Halas, Chem. Phys. Lett. 288(2-4), 243-247 (1998).

${ }^{4}$ S. Link and M. A. El-Sayed, J. Phys. Chem. B 103(40), 8410-8426 (1999).

${ }^{5}$ R. Jin, Y. W. Cao, C. A. Mirkin, K. L. Kelly, G. C. Schatz, and J. G. Zheng, Science 294(5548), 1901-1903 (2001).

${ }^{6}$ G. C. Schatz, Acc. Chem. Res. 17(10), 370-376 (1984).

${ }^{7}$ K. A. Willets and R. P. Van Duyne, Annu. Rev. Phys. Chem. 58, 267-297 (2007).

${ }^{8}$ S. Eustisa and M. A. El-Sayed, Chem. Soc. Rev. 35(3), 209-217 (2006).

${ }^{9}$ D. P. Fromm, A. Sundaramurthy, P. J. Schuck, G. Kino, and W. E. Moerner, Nano Lett. 4(5), 957-961 (2004).

${ }^{10}$ K.-H. Su, Q.-H. Wei, X. Zhang, J. J. Mock, D. R. Smith, and S. Schultz, Nano Lett. 3(8), 1087-1090 (2003).

${ }^{11}$ R. Chikkaraddy, B. de Nijs, F. Benz, S. J. Barrow, O. A. Scherman, E. Rosta, A. Demetriadou, P. Fox, O. Hess, and J. J. Baumberg, Nature 535, 127-130 (2016).

${ }^{12}$ H. Xu, J. Aizpurua, M. Käll, and P. Apell, Phys. Rev. E 62(3), 4318-4324 (2000).

${ }^{13}$ E. Hao and G. C. Schatz, J. Chem. Phys. 120(1), 357-366 (2004).

${ }^{14}$ Y. Sawai, B. Takimoto, H. Nabika, K. Ajito, and K. Murakoshi, J. Am. Chem. Soc. 129(6), 1658-1662 (2007).

${ }^{15}$ T. Konishi, M. Kiguchi, M. Takase, F. Nagasawa, H. Nabika, K. Ikeda, K. Uosaki, K. Ueno, H. Misawa, and K. Murakoshi, J. Am. Chem. Soc. 135(3), 1009-1014 (2012).

${ }^{16}$ C. Dahmen, B. Schmidt, and G. von Plessen, Nano Lett. 7(2), 318-322 (2007).

${ }^{17}$ E. Prodan, C. Radloff, N. J. Halas, and P. Nordlander, Science 302(5644), 419422 (2003).

${ }^{18}$ P. Nordlander, C. Oubre, E. Prodan, K. Li, and M. I. Stockman, Nano Lett. 4(5), 899-903 (2004). 
${ }^{19}$ X. Shi, K. Ueno, T. Oshikiri, Q. Sun, K. Sasaki, and H. Misawa, Nat. Nanotechnol. 13(10), 953-958 (2018).

${ }^{20}$ J. Yang, Q. Sun, K. Ueno, X. Shi, T. Oshikiri, H. Misawa, and Q. Gong, Nat. Commun. 9, 4858-1-4858-8 (2018).

${ }^{21}$ M.-W. Chu, V. Myroshnychenko, C. H. Chen, J.-P. Deng, C.-Y. Mou, and F. J. G. de Abajo, Nano Lett. 9(1), 399-404 (2008).

${ }^{22}$ S.-C. Yang, H. Kobori, C.-L. He, M.-H. Lin, H.-Y. Chen, C. Li, M. Kanehara, T. Teranishi, and S. Gwo, Nano Lett. 10(2), 632-637 (2010).

${ }^{23}$ Q. Sun, H. Yu, K. Ueno, A. Kubo, Y. Matsuo, and H. Misawa, ACS Nano 10(3), 3835-3842 (2016).

${ }^{24}$ K. Ueno, J. Yang, Q. Sun, D. Aoyo, H. Yu, T. Oshikiri, A. Kubo, Y. Matsuo, Q. Gong, and H. Misawa, Appl. Mater. Today 14, 159-165 (2019).

${ }^{25}$ J. A. Fan, C. Wu, K. Bao, J. Bao, R. Bardhan, N. J. Halas, V. N. Manoharan, P. Nordlander, G. Shvets, and F. Capasso, Science 328(5982), 1135-1138 (2010).

${ }^{26}$ J. B. Lassiter, H. Sobhani, J. A. Fan, J. Kundu, F. Capasso, P. Nordlander, and N. J. Halas, Nano Lett. 10(8), 3184-3189 (2019).

${ }^{27} \mathrm{H}$. Yu, Q. Sun, J. Yang, K. Ueno, T. Oshikiri, A. Kubo, Y. Matsuo, Q. Gong, and H. Misawa, Opt. Express 25(6), 6883-6894 (2017).

${ }^{28}$ K. Ueno, S. Juodkazis, M. Mino, V. Mizeikis, and H. Misawa, J. Phys. Chem. C 111(11), 4180-4184 (2007).

${ }^{29}$ N. R. Jana, L. Gearheart, and C. J. Murphy, J. Phys. Chem. B 105(19), 4065-4067 (2001).
${ }^{30}$ B. Nikoobakht and M. A. El-Sayed, Chem. Mater. 15(10), 1957-1962 (2003).

${ }^{31}$ X. Ye, L. Jin, H. Caglayan, J. Chen, G. Xing, C. Zheng, V. Doan-Nguyen, Y. Kang, N. Engheta, C. R. Kagan, and C. B. Murray, ACS Nano 6(3), 2804-2817 (2012).

${ }^{32}$ P. B. Johnson and R. W. Christy, Phys. Rev. B 6(12), 4370-4379 (1972).

${ }^{33}$ K. Ueno and H. Misawa, Phys. Chem. Chem. Phys. 15(12), 4093-4099 (2013).

${ }^{34}$ Y. Yokota, K. Ueno, S. Juodkazis, V. Mizeikis, N. Murazawa, H. Misawa, H. Kasa, K. Kintaka, and J. Nishii, J. Photochem. Photobiol. A: Chem 207(1), 126-134 (2009).

${ }^{35}$ Y. Yokota, K. Ueno, and H. Misawa, Chem. Commun. 47(12), 3505-3507 (2011).

${ }^{36}$ J. Jiang, L. Ou-Yang, L. Zhu, J. Zou, and H. Tang, Sci. Rep. 4, 3942-1-3942-9 (2014).

${ }^{37}$ K. Ueno, S. Juodkazis, V. Mizeikis, K. Sasaki, and H. Misawa, J. Am. Chem. Soc. 128(44), 14226-14227 (2006).

${ }^{38}$ K. Ueno, S. Juodkazis, V. Mizeikis, K. Sasaki, and H. Misawa, Adv. Mater. 20(1), 26-30 (2008)

${ }^{39} \mathrm{M}$. Inoue and K. Ohtaka, J. Phys. Soc. Jpn. 52, 3853-3864 (1983).

${ }^{40}$ K. Yoshida, T. Itoh, H. Tamaru, V. Biju, M. Ishikawa, and Y. Ozaki, Phys. Rev. B 81, 115406-1-115406-9 (2010).

${ }^{41}$ Y. Yokota, K. Ueno, and H. Misawa, Small 7(2), 252-258 (2011). 